\title{
PET imaging of COVID-19: the target and the number
}

\author{
E. Guedj ${ }^{1,2,3} \cdot$ A. Verger ${ }^{4,5} \cdot$ S. Cammilleri ${ }^{1,2,3}$
}

Received: 31 March 2020 / Accepted: 6 April 2020 / Published online: 17 April 2020

(C) Springer-Verlag GmbH Germany, part of Springer Nature 2020

In the context of COVID-19 worldwide outbreak, first reports are being published on the potential value of PET imaging. A total of 5 highly suspected or confirmed COVID-19 cases explored with ${ }^{18}$ F-FDG PET have been so far described by Qin et al. [1] and Zou and Zhu [2], at the diagnostic step, showing lung hypermetabolic pulmonary ground glass opacities with low-dose CT correspondence, frequently associated to lymph nodes hypermetabolism. More broadly, Deng et al. [3] also argue for the possible ${ }^{18}$ F-FDG PET utility, as a sensitive tool to detect and monitor inflammatory diseases, such as viral pneumonia, monitor disease progression, and treatment outcomes, according to the major goals of precision medicine in which PET imaging is well-known to be crucially involved [4]. On the opposite, Joob and Wiwanitkit have recalled that ${ }^{18} \mathrm{~F}$-FDG PET is still not recommended in infectious pneumonia, and especially warned of the risk of disease spreading in PET departments [5].

Besides these justified arguments, on one hand, the potential interest of PET imaging to better understand and characterize the disease, especially perhaps between the infectious and immune phases of the disease [6], with possibly also the interest of the targeted development of ImmunoPET in this

This article is part of the Topical Collection on Infection and inflammation

E. Guedj

eric.guedj@ap-hm.fr

1 Aix-Marseille Université, CNRS, Ecole Centrale Marseille, UMR 7249, Institut Fresnel, Marseille, France

2 Department of Nuclear Medicine, Assistance Publique des Hôpitaux de Marseille, Timone University Hospital, Marseille, France

3 CERIMED, Aix-Marseille Université, Marseille, France

4 Department of Nuclear Medicine and Nancyclotep Imaging Platform, Université de Lorraine, F-54000 Nancy, France

5 IADI, Université de Lorraine, INSERM U1254, F-54000 Nancy, France indication [7], and on the other hand, the genuine risk of viral propagation, the PET exploration of suspected or positive COVID-19 cases is more simply today unrealistic because of the number of concerned patients. For example, and while the epidemic peak is still supposed not to be reached, 2365 patients have been newly hospitalized in France with a COVID-19 confirmed diagnosis on the single day of March, 26th 2020 (https://www.gouvernement.fr/info-coronavirus/ carte-et-donnees), while our national PET capacity is currently estimated to approximately 2000 exams per day (https://www.sfmn.org/drive/SECRETARIAT\% 20 G E N ER A L / EN Q UETE_A N N UELLE/ EnqueteNationale2019_publicWeb.pdf). We have also to note that these ${ }^{18}$ F-FDG PET exams are associated with more complex logistic and long procedures, in particular for the disinfection between patients. Above all, the feasibility issue has to critically integrate the necessary continuation of management of non-COVID-19 diseases with explorations that cannot be canceled or delayed without causing a loss of chance for the patients, both for acute and chronic diseases, and especially for cancer diagnosis and evaluation.

In this line, targeted explorations based on precision medicine seem hardly compatible with mass exploration of numerous patients without delay and in a very short time period. Besides cutting-edge PET investigations currently applied on selected patients and on specific indications, this contradiction highlights the further need of development of light PET protocols as previously developed in radiology for today the very useful low-dose thoracic CT [8], allowing broader exploration availability with shorter procedures for acquisition duration and perhaps also for the uptake period, but probably by preserving the whole-body exploration to better characterize the extension of the disease and its prognosis. New technological achievements based on ultra-low dose whole-body PET instrumentation [9] combined with deep neural networks for reconstruction including generative adversarial networks [10, 11] constitute a great opportunity for such developments which need to be encouraged, with also ultimately possible larger applications in other contexts for example for cancer screening [12]. 


\section{Compliance with ethical standards}

Conflict of interest The authors declare that they have no conflict of interest.

Research involving human participants and/or animals and informed consent This article does not contain any studies with human participants or animals performed by any of the authors.

\section{References}

1. Qin C, Liu F, Yen TC, Lan X. F-FDG PET/CT findings of COVID19: a series of four highly suspected cases. Eur J Nucl Med Mol Imaging. 2020;47:1281-6. https://doi.org/10.1007/s00259-02004734-w.

2. Zou S, Zhu X. FDG PET/CT of COVID-19. Radiology. 2020;200770. https://doi.org/10.1148/radiol.2020200770.

3. Deng Y, Lei L, Chen Y, Zhang W. The potential added value of FDG PET/CT for COVID-19 pneumonia. Eur J Nucl Med Mol Imaging. 2020. https://doi.org/10.1007/s00259-020-04767-1.

4. Guedj E, Cammilleri S, Verger A. Predictive medicine: towards a multi-parametric imaging for a personal risk stratification. Eur J Nucl Med Mol Imaging. 2017;44:196-8. https://doi.org/10.1007/ s00259-016-3522-2.

5. Joob B, Wiwanitkit V. 18F-FDG PET/CT and COVID-19. Eur J Nucl Med Mol Imaging. 2020. https://doi.org/10.1007/s00259020-04762-6.
6. Fu Y, Cheng Y, Wu Y. Understanding SARS-CoV-2-mediated inflammatory responses: from mechanisms to potential therapeutic tools. Virol Sin. 2020. https://doi.org/10.1007/s12250-020-002074.

7. Fu R, Carroll L, Yahioglu G, Aboagye EO, Miller PW. Antibody fragment and Affibody ImmunoPET imaging agents: radiolabelling strategies and applications. ChemMedChem. 2018;13:2466-78. https://doi.org/10.1002/cmdc.201800624.

8. Kang Z, Li X, Zhou S. Recommendation of low-dose CT in the detection and management of COVID-2019. Eur Radiol. 2020. https://doi.org/10.1007/s00330-020-06809-6.

9. Slomka PJ, Pan T, Germano G. Recent advances and future progress in PET instrumentation. Semin Nucl Med. 2016;46:5-19. https://doi.org/10.1053/j.semnuclmed.2015.09.006.

10. Ouyang J, Chen KT, Gong E, Pauly J, Zaharchuk G. Ultra-low-dose PET reconstruction using generative adversarial network with feature matching and task-specific perceptual loss. Med Phys. 2019;46:3555-64. https://doi.org/10.1002/mp.13626.

11. Schwyzer M, Ferraro DA, Muehlematter UJ, Curioni-Fontecedro A, Huellner MW, von Schulthess GK, et al. Automated detection of lung cancer at ultralow dose PET/CT by deep neural networks initial results. Lung Cancer. 2018;126:170-3. https://doi.org/10. 1016/j.lungcan.2018.11.001.

12. Schaefferkoetter JD, Yan J, Sjöholm T, Townsend DW, Conti M, Tam JK, et al. Quantitative accuracy and lesion detectability of lowdose. J Nucl Med. 2017;58:399-405. https://doi.org/10.2967/ jnumed.116.177592.

Publisher's note Springer Nature remains neutral with regard to jurisdictional claims in published maps and institutional affiliations. 